\title{
Neoadjuvant Chemotherapy for Stage II-III Breast Cancer: A Single-center Experience
}

\section{Haidi Abd El Zaher}

Suez Canal University Faculty of Medicine

\section{Hamdad Fathy}

Suez Canal University Faculty of Medicine

\section{Mohamed Abozeid}

Suez Canal University Faculty of Medicine

Mohammed Faisal ( $\nabla$ m.faisal@med.suez.edu.eg)

Suez Canal University Faculty of Medicine https://orcid.org/0000-0002-2162-1741

\section{Research}

Keywords: Neoadjuvant Chemotherapy, Conservative Breast Surgery, Breast Cancer, Mastectomy

Posted Date: October 25th, 2021

DOl: https://doi.org/10.21203/rs.3.rs-968632/v1

License: (9) This work is licensed under a Creative Commons Attribution 4.0 International License. Read Full License 


\section{Abstract}

Introduction: We conducted this study to reflect a single-center experience with the use of neoadjuvant systemic chemotherapy for the management of women with operable breast cancer.

Methods: We conducted a retrospective chart review on all women presenting with operable, stage II-III, breast cancer and were scheduled for neoadjuvant systemic chemotherapy at Suez Canal University Hospital. The primary outcome of this study was to estimate the proportion of patients with operable breast cancer who become eligible for breast conservative surgery (CBS) after neoadjuvant systemic chemotherapy.

Results: A total of 147 patients were included. Before the initiation of chemotherapy, only 66 (44.9\%) patients were indicated for breast conservative surgery (CBS). A total of 40 (50.6\%) new patients, out of the 81 patients who were ineligible before chemotherapy, became eligible for breast conservative surgery after neoadjuvant chemotherapy $(95 \% \mathrm{Cl} 39.3-61.9 \%)$. On the other hand, nine (13.6\%) patients became ineligible for breast conservative surgery after neoadjuvant chemotherapy. Out of the 98 eligible patients for breast conservative surgery after chemotherapy, 72 (73.5\%) patients underwent the surgery and the remaining 26 patients chose total modified radical mastectomy (MRM). A total of 55 (76.4\%) patients achieved pathological complete response pCR. One woman $(0.1 \%)$ experienced relapse at the $3^{\text {rd }}$ year of follow-up and three women (2\%) experienced relapse at the $5^{\text {th }}$ year of follow-up. The difference between patients who underwent breast conservative surgery and total mastectomy was not statistically significant ( $p=0.22$ and 0.07 , respectively).

Conclusion: Neoadjuvant chemotherapy can play a crucial role in increasing the rate of eligibility for breast conservative surgery among women with operable, stage II-III, breast cancer.

\section{Introduction}

Locally advanced breast cancer ( $L A B C$ ) refers to patients with more than $5 \mathrm{~cm}$ primary tumors involving chest or skin, or to patients with mammary or axillary nodal metastasis (N2/N3 and T3/T4). The initial treatment strategies of surgery, radiation, or both of them had a little impact on survival rates in these patients, hence were changed to involve multimodality approach that includes neoadjuvant chemotherapy in the 1970s [1]. Neoadjuvant chemotherapy has been widely extended to treat early (operable) breast cancer [2].

Neoadjuvant chemotherapy had many advantages over the conventional approach of surgery followed by adjuvant chemotherapy. Considerable evidence has stated that neoadjuvant systemic chemotherapy has improved overall survival (OS) rates and disease free-survival (DFS) [1, 3-5]. It significantly decreases lymph nodal metastasis and primary tumor size in most cases; thus, it can increase the utility of breast-conserving surgery, which improves body appearance and sexual function in comparison to mastectomy [6-10]. A recent meta-analysis, involving 10 studies with 4756 patients, reported that neoadjuvant chemotherapy has increased breast conserving-surgery from $49-65 \%$ compared to adjuvant 
therapy [11]. A recent prospective study, enrolling 634 patients, reported that neoadjuvant systemic therapy facilitated breast conservation surgery in $53.2 \%$ of women with triple-negative breast cancer [12].

Besides, neoadjuvant systemic chemotherapy has provided good results regarding tumor recurrent rates in women with breast cancer. Comparable breast cancer recurrence rates were reported between the preoperative $(7.9 \%)$ and postoperative chemotherapy groups (5.8\%) [13]. About $27 \%$ of patients who were appropriate for mastectomy underwent breast conservation surgery and their breast cancer recurrence rates were $14.3 \%$. The breast cancer recurrence rates of patients appropriate for breast conservation surgery at the outset were $6.9 \%$. Both groups had comparable rates of overall survival and disease-free survival. A study by Chen et al enrolled 340 women with breast conservation surgery following preoperative systemic chemotherapy and reported $5 \%$ of 5-year ipsilateral breast cancer recurrence rate and $9 \%$ of locoregional recurrence [4]. Another study affirmed the value of neoadjuvant chemotherapy and the role of preoperative Taxanes in increasing response rates in premenopausal women with breast cancer [3].

We conducted this study to investigate the importance of neoadjuvant systemic chemotherapy for the management of women with operable, non-inflammatory, stage II and III breast cancer in Suez Canal University Hospital.

\section{Materials And Methods}

The protocol of the current study was approved by the local ethics committee of Suez Canal University Hospital (\#3412). The need for signed informed consent was waived due to the retrospective nature of the study. All procedures run in compliance with the standards of the Declaration of Helsinki [14]. We prepared the following manuscript in concordance with STROBE guidelines [15].

\subsection{Study design and Patients:}

We conducted a retrospective chart review on all women presenting with operable, stage II-III, breast cancer and were scheduled for neoadjuvant systemic treatment at Suez Canal University Hospital through the period from January 2019 to February 2021. Only women whose breast cancer diagnosis was confirmed with a biopsy and who were treatment-naïve were included. We excluded records of the patients with inflammatory disease and patients who did not receive a full course of neoadjuvant systemic treatment due to progression.

\subsection{Study's Procedures:}

We collected the baseline data of the eligible patients, which included demographics characteristics, examination findings, the findings of breast imaging, Echocardiography, Chest $\mathrm{X}$ ray, Pelviabdominal ultrasound, immunohistochemical results, the eligibility for surgery, and the pre-chemotherapy type of surgery. At our center, a surgical specialist determines the eligibility for breast conservative surgery according to the tumor size, multi-centricity, presence of diffuse calcification, the presence of nipple 
invasion or tumors located close to the nipple, and the expected cosmetic result. All women who were deemed eligible for surgery received neoadjuvant systemic treatment. The regimen was four cycles of doxorubicin/cyclophosphamide, followed by four cycles of Paclitaxel for all participants, with trastuzumab for patients with human epidermal growth factor receptor 2 (HER2)-positive breast cancer. For patients with triple-negative breast cancer (TNBC), we added carboplatin to Paclitaxel. Then, breast imaging was performed after the completion of the regimen's cycles. Six weeks later, breast cancer surgery was performed. Breast cancer surgery was performed after an informed discussion between the treating surgeon and patients. The choice of breast conservative surgery or total mastectomy was based on surgeon/patient's decision or when the breast conservative surgery deemed unsuccessful intraoperatively.

\subsection{Study's Outcomes:}

The primary outcome of this study was to estimate the proportion of patients with operable breast cancer who become eligible for breast conservative surgery after neoadjuvant systemic chemotherapy. The choice of post-chemotherapy surgery was based on the surgeon/patient's decision or when the breast conservative surgery was deemed unsuccessful intraoperatively. The secondary outcomes of this study were the rate of complete pathological response (PCR) after breast conservative surgery and the rate of recurrence after surgery.

\subsection{Statistical analysis:}

We employed descriptive statistics to describe the patients' age, menopausal status, stage, grade, immunohistochemical results, the pre-chemotherapy type of surgery, contradictions for breast conservative surgery, type of chemotherapy, the post-chemotherapy contradictions for breast conservative surgery, the success rate of breast conservative surgery, and recurrence after breast conservative surgery. The proportion of the patients with operable breast cancer who become eligible for breast conservative surgery after neoadjuvant systemic chemotherapy was calculated with $95 \%$ confidence intervals $(\mathrm{Cl})$ using exact binomial methods. Retrieved data were processed with IBM SPSS statistical software (version 25).

\section{Results}

A total of 147 patients were included, with a mean age of $47.7 \pm 10.5$ years old. Of them, $45(30.6 \%)$ were post-menopause. The most commonly encountered pathological grade was stage III (49.7\%), followed by stage II (38.8\%). Invasive ductal carcinoma accounted for the vast majority of the cases $(89.8 \%)$. Concerning the TNM stage, the T2NXM0 (43.5\%) and T3NXM0 (25.9\%) stages were the most common stages. Nearly two-thirds of the patients were estrogen-receptor (ER)-positives, $42.2 \%$ were progesterone receptor (PR)-positive, and $36.7 \%$ of the patients were HER-2 positive. Nearly $59 \%$ of the cases had a KI67 index $>10$ (Table 1). 
Table 1

Preoperative characteristics of the studied group

\begin{tabular}{|c|c|}
\hline Variables & Patients ( $n=147$ ) \\
\hline Age in year, mean $\pm S D$ & $47.7 \pm 10.5$ \\
\hline \multicolumn{2}{|c|}{ Menopausal status, No. (\%) } \\
\hline - Premenopausal & $102(69.4 \%)$ \\
\hline - Postmenopausal & $45(30.6 \%)$ \\
\hline \multicolumn{2}{|c|}{ Pathological Grade, No. (\%) } \\
\hline - Grade I & $14(9.5 \%)$ \\
\hline - Grade II & $57(38.8 \%)$ \\
\hline - Grade III & $73(49.7 \%)$ \\
\hline - Unknown Grade & $3(2 \%)$ \\
\hline \multicolumn{2}{|l|}{ Histological Type, No. (\%) } \\
\hline - Ductal & $132(89.8 \%)$ \\
\hline - Lobular & $4(2.7 \%)$ \\
\hline - Invasive, Nos & $9(6.1 \%)$ \\
\hline - Other & $2(1.4 \%)$ \\
\hline \multicolumn{2}{|l|}{ TNM Stage, No. (\%) } \\
\hline - T2NO & $12(8.2 \%)$ \\
\hline - T3NO & $3(2 \%)$ \\
\hline - T4NO & $6(4.1 \%)$ \\
\hline$-\mathrm{T} 1 \mathrm{NX}$ & $7(4.8 \%)$ \\
\hline$-\mathrm{T} 2 \mathrm{NX}$ & $64(43.5 \%)$ \\
\hline - T3NX & $38(25.9 \%)$ \\
\hline - T4NX & $17(11.6 \%$ \\
\hline \multicolumn{2}{|c|}{ Hormonal Receptors, No. (\%) } \\
\hline - ER positive & $94(63.9 \%)$ \\
\hline - PR Positive & $62(42.2 \%)$ \\
\hline - Her-2 positive & $54(36.7 \%)$ \\
\hline KI67 index, No. (\%) & \\
\hline
\end{tabular}




\begin{tabular}{|ll|}
\hline Variables & Patients $(\mathbf{n}=\mathbf{1 4 7})$ \\
\hline$-<10$ & $45(41.5 \%)$ \\
$->10$ & $86(58.5 \%)$ \\
\hline
\end{tabular}

Before the initiation of chemotherapy, only 66 (44.9\%) patients were indicated for breast conservative surgery (Figure 1). The most common contraindications for breast conservative surgery were large tumor size (48.1\%), multi-centricity (18.5\%), and poor cosmetic outcome (14.8\%). The distribution of chemotherapeutic regimens was as the following: Anthracyclins plus Taxanes (38.1\%), anthracyclines plus taxanes plus trastuzumab (36.7\%), and anthracyclines plus taxanes plus carboplatin (25.2\%). After the completion of the chemotherapy regimen, $98(66.7 \%)$ of the women were deemed eligible for breast conservative surgery (Figure 1). A total of $40(50.6 \%)$ new patients, out of the 81 patients who were ineligible before chemotherapy, became eligible for breast conservative surgery after neoadjuvant chemotherapy ( $95 \% \mathrm{Cl} 39.3-61.9 \%)$. On the other hand, nine (13.6\%) patients, who were eligible before chemotherapy, became ineligible for breast conservative surgery after neoadjuvant chemotherapy. Out of the 98 eligible patients for breast conservative surgery after chemotherapy, 72 (73.5\%) patients underwent the surgery and the remaining 26 patients chose total mastectomy (Table 2). On the other hand, the causes of refusing breast conservative surgery in the 49 ineligible patients were large tumor size $(14.3 \%$ ) or high recurrence risk (diffuse micro-calcifications or multi-centricity $=81.6 \%$ ), or patients' choice (4.1\%). Out of the 72 patients who underwent conservative surgery, a total of $55(76.4 \%)$ patients were found to have $\mathrm{PCR}$.

Table 2

Post-chemotherapy characteristics of the studied group

\begin{tabular}{|lllll|}
\hline & $\begin{array}{l}\text { Total (n } \\
\text { =147) }\end{array}$ & $\begin{array}{l}\text { Remained } \\
\text { BCS }\end{array}$ & $\begin{array}{l}\text { Converted to } \\
\text { BCS }\end{array}$ & $\begin{array}{l}\text { Converted to } \\
\text { Mastectomy }\end{array}$ \\
\hline $\begin{array}{l}\text { BCS candidate pre- } \\
\text { chemotherapy }\end{array}$ & $66(44.9 \%)$ & $57(86.4 \%)$ & - & $9(13.6 \%)$ \\
\hline $\begin{array}{l}\text { BCS ineligible before } \\
\text { chemotherapy }\end{array}$ & $81(55.1 \%)$ & - & $40(50.6 \%)$ & - \\
\hline Final surgery decision & & & & \\
\hline - BCS & $98(65.9 \%)$ & $72(73.5 \%)$ & - & $26(26.5 \%)$ \\
\hline - Mastectomy & $49(34.1 \%)$ & - & - & - \\
\hline
\end{tabular}

Overall, one woman $(0.1 \%)$ experienced recurrence at the 3rd year of follow-up and three women ( $2 \%)$ experienced recurrence at the 5th year of follow-up. The difference between patients who underwent breast conservative surgery and total mastectomy was not statistically significant ( $p=0.22$ and 0.07 , respectively). 
Patients, who were deemed eligible for breast conservative surgery after chemotherapy, were significantly older than ineligible patients $(p=0.019)$. Ineligible patients were more likely to have higher grade $(p$ $=0.007)$, higher TNMA stage $(p<0.001)$, HER-2 negative $(p=0.004)$, higher KI67 index $(p=0.001)$, larger tumor size $(p=0.001)$, more diffuse micro-calcification $(p=0.001)$, and were more likely to receive anthracyclins plus taxanes only $(p=0.008)$. Besides, $p C R$ significantly increased the rate of eligible patients for breast conservative surgery.

\section{Discussion}

The uses of neoadjuvant chemotherapy in the setting of breast cancer have expanded widely over the past decade. Although the benefits of neoadjuvant chemotherapy in reducing tumor size appear to be well-recognized [16], little is known about the real-world experience with the indications and outcomes of neoadjuvant chemotherapy in developing countries, like Egypt. In this study, we aimed to present an Egyptian center experience regarding the use of neoadjuvant systemic chemotherapy for the management of women with operable, non-inflammatory, stage II and III breast cancer. Our results indicated that the neoadjuvant chemotherapy led to a notable increase in the proportion of breast cancer patients who performed breast conservative surgery. Besides, more than two-thirds of the patients, who underwent breast conservative surgery, achieved $\mathrm{pCR}$. The conversion of the patients to breast conservative surgery after neoadjuvant chemotherapy did not have a negative impact on the recurrence rate as well. The comparative analysis showed that patients who responded to neoadjuvant chemotherapy were more likely to be younger, HER-2 positive, and had lower tumor grade and stage.

Breast conservative surgery is the modality of choice for early breast cancer (stage I-II) due to its comparable survival benefits and better cosmetic outcome, compared to radical surgery [17]. In patients with more advanced carcinoma, a relatively lower proportion of the patients become eligible for breast conservative surgery, who are usually young patients with small localized tumor and favorable physical status [18]. Neoadjuvant chemotherapy has the advantages of downstaging tumor size before surgery and assessing treatment response. In return, neoadjuvant chemotherapy can permit less-invasive surgery, better cosmetic outcomes, and less risk of postoperative lymphedema. An additional advantage of neoadjuvant chemotherapy is its ability to aid intraoperative tumor recognition and reduce the possibility of extensive residual disease $[19,20]$. In light of the aforementioned benefits of neoadjuvant chemotherapy, several studies have assessed the efficacy in increasing the rate of breast cancer patients eligible for conservative surgery. In this report, we found that the use of neoadjuvant chemotherapy increased the proportion of women eligible for breast conservative surgery by $50 \%$. In line with these findings, Debled et al. [21], noted that $71 \%$ of HER2-positive patients underwent breast conservative surgery after neoadjuvant chemotherapy. The same results were obtained by Semiglazov et al. [21], Vergine et al. [22], and Cho et al [23]. In Golshan et al. [24], $42 \%$ of patients who were initially deemed ineligible were converted by neoadjuvant chemotherapy to breast conservative surgery. In a previous meta-analysis, the neoadjuvant chemotherapy led to breast conservative surgery in nearly $65 \%$ of the patients [11]. However, the current literature shows controversy regarding the positive impact of neoadjuvant chemotherapy on the rate of breast conservative surgery. According to Boughey et al. [25], 
the neoadjuvant chemotherapy did not increase the rate of conservative surgery among women with invasive breast cancer.

Higher pCR can intuitively favor the decision of performing breast conservative surgery, in patients who were previously candidates for mastectomy [26]. In this regard, the emergence of taxanes, and other novel agents, has dramatically improved the pathological response following neoadjuvant chemotherapy and, hence, improved the clinical outcomes of the patients [27]. In this study, we found that, out of the 72 patients who underwent conservative surgery, a total of $55(76.4 \%)$ patients were found to have pCR. Such findings may reflect that downsizing the tumor can increase the chance of performing breastconservative surgery without compromising the oncological outcomes. Previously, it was found that patients with PCR had better survival outcomes than patients without pCR and underwent breastconservative surgery [23].

Local recurrence is a major concern in patients undergoing breast-conservative surgery, previous reports indicated that up to $15 \%$ of the patients undergoing conservative surgery will develop locoregional recurrence [28]. However, the application of neoadjuvant chemotherapy can potentially reduce the risk of recurrence. In a recent meta-analysis, the rate of recurrence in patients received neoadjuvant chemotherapy and breast-conservative surgery was $9.2 \%$, compared to $8.3 \%$ in the mastectomy group [29]. In the present study, we found that the 5-year local recurrence-free rate was $98 \%$; the difference between patients who underwent breast conservative surgery and total mastectomy was not statistically significant. Our findings run in line with the results of NSABP-B18 trial [7] and other studies [3,30]. The

study by Ishitobi et al. [31], also found no significant difference in local recurrence-free rate according to type of surgery after neoadjuvant chemotherapy in patients with a planned mastectomy at the initial exam.

We acknowledge the existence of certain limitations of the present study. The current study was a retrospective chart review, with the inherited limitations of misclassifications, missing data, or recording errors. Besides, the results of genetic testing were not available for all patients, which might have affected the choice of surgery. There were no clear data regarding the causes underlying patients' choice to undergo total mastectomy, despite being eligible for conservative surgery. The choice of surgery was based mainly on the surgeon's decision, which may introduce biases in the decision-making process. Lastly, all patients underwent breast magnetic resonance imaging (MRI) after neoadjuvant chemotherapy, which could have increased the rate of mastectomy in the present study; previous studies reported that MRI was a major factor for the increased rate of mastectomy due to its ability to detect additional abnormalities [32].

\section{Conclusion}

Neoadjuvant chemotherapy can play a crucial role in increasing the rate of eligibility for breast conservative surgery among women with operable, stage II-III, breast cancer. Our findings indicated that employing neoadjuvant chemotherapy can increase the rate of eligible breast conservative surgery by 
nearly $50 \%$. The pCR after breast conservative surgery in our study was notably high. Besides, we also found that the use of breast conservative surgery after the neoadjuvant chemotherapy did not significantly increase the risk of recurrence. Given the scarcity in the published literature, it is recommended to conduct rigorous trials, with a large sample size and multinational collaboration, to reflect the clinical usefulness of neoadjuvant chemotherapy in the setting of operable breast cancer.

\section{Abbreviations}

BCS: breast-conservative surgery

BC: Breast cancer.

Cl: Confidence Intervals.

DFS: Disease Free-Survival.

HER-2: Human Epidermal Growth Factor Receptor 2.

MRI: Magnetic Response Imaging.

MRM: Modified Radical Mastectomy.

OS: Overall Survival.

PCR: pathological Complete Response.

STROBE: Strengthening the Reporting of Observational Studies in Epidemiology

TNBC: Triple-Negative Breast Cancer

\section{Declarations}

\section{Ethics approval and consent to participate:}

Official approvals of the responsible ethics committee were gained from Suez Canal University Hospitals (\#3412) in their meeting 12 September 2018.

Consent for publication:

Not applicable

Availability of data and materials:

Not applicable

Competing interests: 
Authors declare that they have no competing interests

\section{Funding:}

Authors declare that they have no funding

\section{Authors' contributions:}

Study conception and design: MF, HA and HF

Acquisition of data: HA, OA, MF

Analysis and interpretation of data: HF, OA and MF

Drafting of manuscript: HA and MF

Critical revision of manuscript: $\mathrm{HA}, \mathrm{OA}, \mathrm{HF}, \mathrm{MF}$.

All authors have read and approved the manuscript.

\section{Acknowledgment:}

None

\section{References}

1. Singletary SE. Neoadjuvant chemotherapy in the treatment of stage II and III breast cancer. Am. J. Surg., vol. 182, Am J Surg; 2001, p. 341-6. https://doi.org/10.1016/S0002-9610(01)00724-3.

2. Buchholz TA, Hunt KK, Whitman GJ, Sahin AA, Hortobagyi GN. Neoadjuvant chemotherapy for breast carcinoma: Multidisciplinary considerations of benefits and risks. Cancer 2003;98:1150-60. https://doi.org/10.1002/cncr.11603.

3. Rastogi P, Anderson SJ, Bear HD, Geyer CE, Kahlenberg MS, Robidoux A, et al. Preoperative chemotherapy: Updates of national surgical adjuvant breast and bowel project protocols B-18 and B27. J Clin Oncol 2008;26:778-85. https://doi.org/10.1200/JC0.2007.15.0235.

4. Chen AM, Meric-Bernstam F, Hunt KK, Thames HD, Oswald MJ, Outlaw ED, et al. Breast conservation after neoadjuvant chemotherapy: The M.D. Anderson cancer center experience. J Clin Oncol 2004;22:2303-12. https://doi.org/10.1200/JC0.2004.09.062.

5. Bear HD, Anderson S, Smith RE, Geyer CE, Mamounas EP, Fisher B, et al. Sequential preoperative or postoperative docetaxel added to preoperative doxorubicin plus cyclophosphamide for operable breast cancer: National surgical adjuvant breast and bowel project protocol B-27. J Clin Oncol 2006;24:2019-27. https://doi.org/10.1200/JC0.2005.04.1665.

6. Fisher B, Brown A, Mamounas E, Wieand S, Robidoux A, Margolese RG, et al. Effect of preoperative chemotherapy on local-regional disease in women with operable breast cancer: Findings from 
national surgical adjuvant breast and bowel project B-18. J Clin Oncol 1997;15:2483-93. https://doi.org/10.1200/JC0.1997.15.7.2483.

7. Wolmark N, Wang J, Mamounas E, Bryant J, Fisher B. Preoperative chemotherapy in patients with operable breast cancer: nine-year results from National Surgical Adjuvant Breast and Bowel Project B-18. J Natl Cancer Inst Monogr 2001:96-102. https://doi.org/10.1093/oxfordjournals.jncimonographs.a003469.

8. Van der Hage JA, Van de Velde CJH, Julien JP, Tubiana-Hulin M, Vandervelden C, Duchateau L. Preoperative chemotherapy in primary operable breast cancer: Results from the European Organization for Research and Treatment of Cancer Trial 10902. J Clin Oncol 2001;19:4224-37. https://doi.org/10.1200/JC0.2001.19.22.4224.

9. Hopwood P, Fletcher I, Lee A, Al Ghazal S. A body image scale for use with cancer patients. Eur J Cancer 2001;37:189-97. https://doi.org/10.1016/S0959-8049(00)00353-1.

10. Thirlaway K, Fallowfield L, Cuzick J. The sexual activity questionnaire: A measure of women's sexual functioning. Qual Life Res 1996;5:81-90. https://doi.org/10.1007/BF00435972.

11. Asselain B, Barlow W, Bartlett J, Bergh J, Bergsten-Nordström E, Bliss J, et al. Long-term outcomes for neoadjuvant versus adjuvant chemotherapy in early breast cancer: meta-analysis of individual patient data from ten randomised trials. Lancet Oncol 2018;19:27-39. https://doi.org/10.1016/S1470-2045(17)30777-5.

12. Golshan M, Loibl S, Wong SM, Houber JB, O’Shaughnessy J, Rugo HS, et al. Breast Conservation after Neoadjuvant Chemotherapy for Triple-Negative Breast Cancer: Surgical Results from the BrighTNess Randomized Clinical Trial. JAMA Surg 2020;155:195410. https://doi.org/10.1001/jamasurg.2019.5410.

13. Fisher B, Bryant J, Wolmark N, Mamounas E, Brown A, Fisher ER, et al. Effect of preoperative chemotherapy on the outcome of women with operable breast cancer. J Clin Oncol 1998;16:267285. https://doi.org/10.1200/JC0.1998.16.8.2672.

14. JAVA. Declaration of Helsinki World Medical Association Declaration of Helsinki. Bull World Heal Organ 2013;79:373-4. https://doi.org/S0042-96862001000400016 [pii].

15. Moher D, Hopewell S, Schulz KF, Montori V, Gøtzsche PC, Devereaux PJ, et al. CONSORT 2010 explanation and elaboration: Updated guidelines for reporting parallel group randomised trials. Int J Surg 2012. https://doi.org/10.1016/j.ijsu.2011.10.001.

16. Masood S. Neoadjuvant chemotherapy in breast cancers. Women's Heal 2016;12:480-91. https://doi.org/10.1177/1745505716677139.

17. Cheng H, Clymer JW, Ferko NC, Patel L, Soleas IM, Cameron CG, et al. A systematic review and metaanalysis of harmonic technology compared with conventional techniques in mastectomy and breastconserving surgery with lymphadenectomy for breast cancer. Breast Cancer Targets Ther 2016;8:125-40. https://doi.org/10.2147/BCTT.S110461.

18. Almahariq MF, Quinn TJ, Siddiqui Z, Jawad MS, Chen PY, Gustafson GS, et al. Breast conserving therapy is associated with improved overall survival compared to mastectomy in early-stage, lymph 
node-negative breast cancer. Radiother Oncol 2020;142:186-94.

https://doi.org/10.1016/j.radonc.2019.09.018.

19. Charfare H, Limongelli S, Purushotham AD. Neoadjuvant chemotherapy in breast cancer. Br J Surg 2005;92:14-23. https://doi.org/10.1002/bjs.4840.

20. Selli C, Sims AH. Neoadjuvant Therapy for Breast Cancer as a Model for Translational Research. Breast Cancer Basic Clin Res 2019;13. https://doi.org/10.1177/1178223419829072.

21. Debled M, Macgrogan G, Breton-Callu C, Ferron S, Hurtevent G, Fournier M, et al. Surgery following neoadjuvant chemotherapy for HER2-positive locally advanced breast cancer. Time to reconsider the standard attitude. Eur J Cancer 2015;51:697-704. https://doi.org/10.1016/j.ejca.2015.01.063.

22. Vergine M, Scipioni P, Garritano S, Colangelo M, Di Paolo A, Livadoti G, et al. Breast-conserving surgery after neoadjuvant chemotherapy in patients with locally advanced cancer. Preliminary results. G Di Chir 2013;34:254-6. https://doi.org/10.11138/gchir/2013.34.9.254.

23. Cho JH, Park JM, Park HS, Park S, Kim S II, Park BW. Oncologic safety of breast-conserving surgery compared to mastectomy in patients receiving neoadjuvant chemotherapy for locally advanced breast cancer. J Surg Oncol 2013;108:531-6. https://doi.org/10.1002/jso.23439.

24. Golshan M, Cirrincione CT, Sikov WM, Berry DA, Jasinski S, Weisberg TF, et al. Impact of neoadjuvant chemotherapy in stage II-III triple negative breast cancer on eligibility for breast-conserving surgery and breast conservation rates: Surgical results from CALGB 40603 (Alliance). Ann. Surg., vol. 262, Lippincott Williams and Wilkins; 2015, p. 434-8. https://doi.org/10.1097/SLA.0000000000001417.

25. Boughey JC, Wagner J, Garrett BJ, Harker L, Middleton LP, Babiera G V., et al. Neoadjuvant chemotherapy in invasive lobular carcinoma may not improve rates of breast conservation. Ann Surg Oncol 2009;16:1606-11. https://doi.org/10.1245/s10434-009-0402-z.

26. Criscitiello C, Golshan M, Barry WT, Viale G, Wong S, Santangelo M, et al. Impact of neoadjuvant chemotherapy and pathological complete response on eligibility for breast-conserving surgery in patients with early breast cancer: A meta-analysis. Eur J Cancer 2018;97:1-6. https://doi.org/10.1016/j.ejca.2018.03.023.

27. Masood S. Neoadjuvant chemotherapy in breast cancers. Women's Heal 2016;12:480-91. https://doi.org/10.1177/1745505716677139.

28. Bartelink H, Horiot J-C, Poortmans P, Struikmans H, Van den Bogaert W, Barillot I, et al. Recurrence Rates after Treatment of Breast Cancer with Standard Radiotherapy with or without Additional Radiation. N Engl J Med 2001;345:1378-87. https://doi.org/10.1056/nejmoa010874.

29. Zhou X, Li Y. Local Recurrence after Breast-Conserving Surgery and Mastectomy Following Neoadjuvant Chemotherapy for Locally Advanced Breast Cancer-a Meta-Analysis. Breast Care 2016;11:345-51. https://doi.org/10.1159/000450626.

30. Touboul E, Lefranc JP, Blondon J, Buffat L, Deniaud E, Belkacémi Y, et al. Primary chemotherapy and preoperative irradiation for patients with stage II larger than $3 \mathrm{~cm}$ or locally advanced noninflammatory breast cancer. Radiother Oncol 1997;42:219-29. https://doi.org/10.1016/S01678140(97)01923-3. 
31. Ishitobi M, Ohsumi S, Inaji H, Ohno S, Shigematsu H, Akiyama F, et al. Ipsilateral breast tumor recurrence (IBTR) in patients with operable breast cancer who undergo breast-conserving treatment after receiving neoadjuvant chemotherapy: Risk factors of IBTR and validation of the MD Anderson Prognostic Index. Cancer 2012;118:4385-93. https://doi.org/10.1002/cncr.27377.

32. Mukhtar RA, Yau C, Rosen M, Tandon VJ, Hylton N, Esserman LJ. Clinically Meaningful Tumor Reduction Rates Vary by Prechemotherapy MRI Phenotype and Tumor Subtype in the I-SPY 1 TRIAL (CALGB 150007/150012; ACRIN 6657). Ann Surg Oncol 2013;20:3823-30. https://doi.org/10.1245/s10434-013-3038-y.

\section{Figures}

\section{BCS Indication}

$80.00 \%$

$70.00 \%$

$66.70 \%$

$60.00 \%$
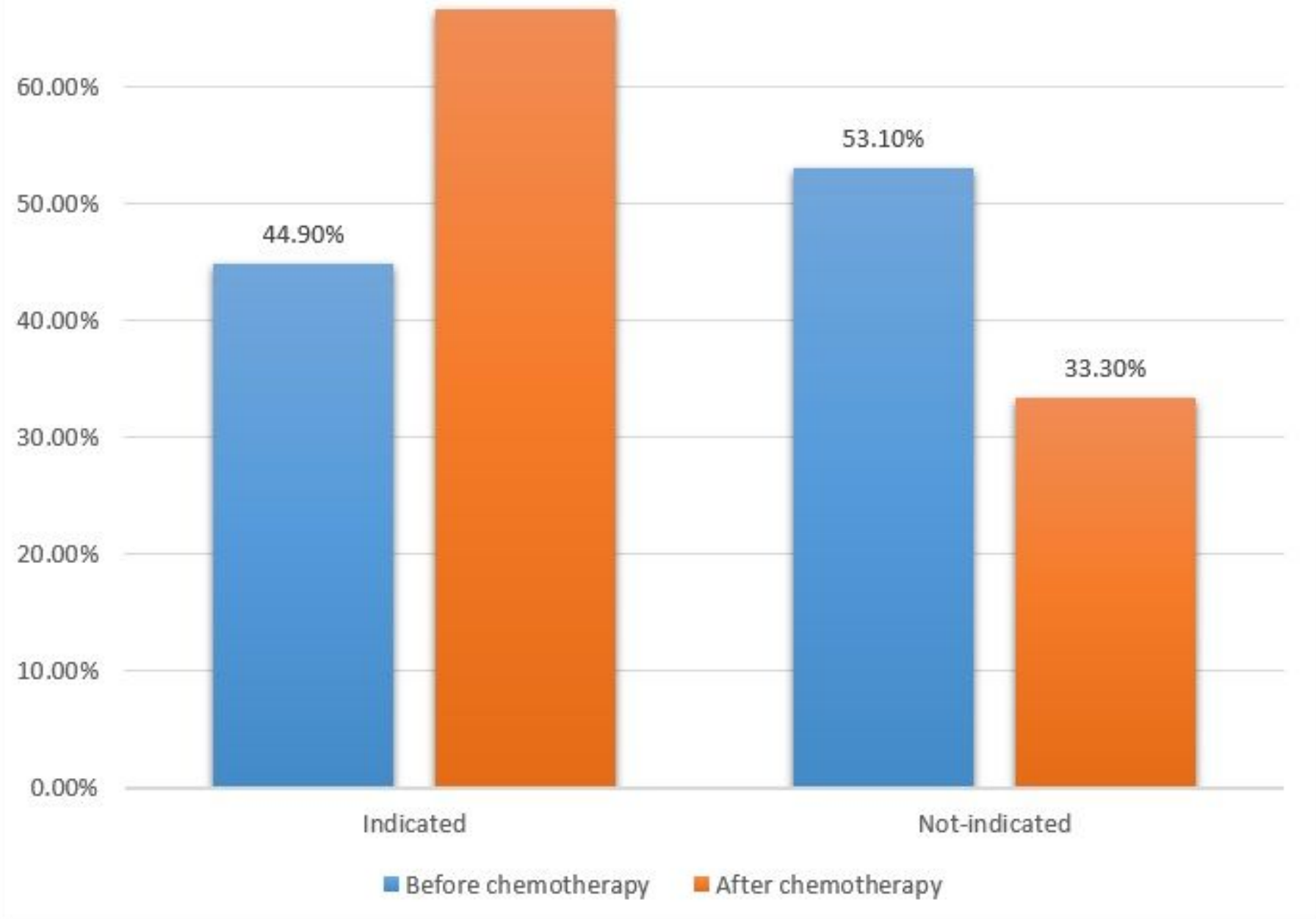


\section{Figure 1}

The frequency of patients indicated for BCS before and after chemotherapy 